\title{
CD44 cell adhesion molecules
}

\author{
S Goodison, V Urquidi, D Tarin
}

\begin{abstract}
The CD44 proteins form a ubiquitously expressed family of cell surface adhesion molecules involved in cell-cell and cellmatrix interactions. The multiple protein isoforms are encoded by a single gene by alternative splicing and are further modified by a range of post-translational modifications. CD44 proteins are single chain molecules comprising an N-terminal extracellular domain, a membrane proximal region, a transmembrane domain, and a cytoplasmic tail. The CD44 gene has only been detected in higher organisms and the amino acid sequence of most of the molecule is highly conserved between mammalian species. The principal ligand of CD44 is hyaluronic acid, an integral component of the extracellular matrix. Other CD44 ligands include osteopontin, serglycin, collagens, fibronectin, and laminin. The major physiological role of CD44 is to maintain organ and tissue structure via cell-cell and cell-matrix adhesion, but certain variant isoforms can also mediate lymphocyte activation and homing, and the presentation of chemical factors and hormones. Increased interest has been directed at the characterisation of this molecule since it was observed that expression of multiple CD44 isoforms is greatly upregulated in neoplasia. CD44, particularly its variants, may be useful as a diagnostic or prognostic marker of malignancy and, in at least some human cancers, it may be a potential target for cancer therapy. This review describes the structure of the CD44 gene and discusses some of its roles in physiological and pathological processes.

(F Clin Pathol: Mol Pathol 1999;52:189-196)
\end{abstract}

Keywords: CD44 structure; hyaluronic acid; alternative splicing; neoplasia

Standard exons

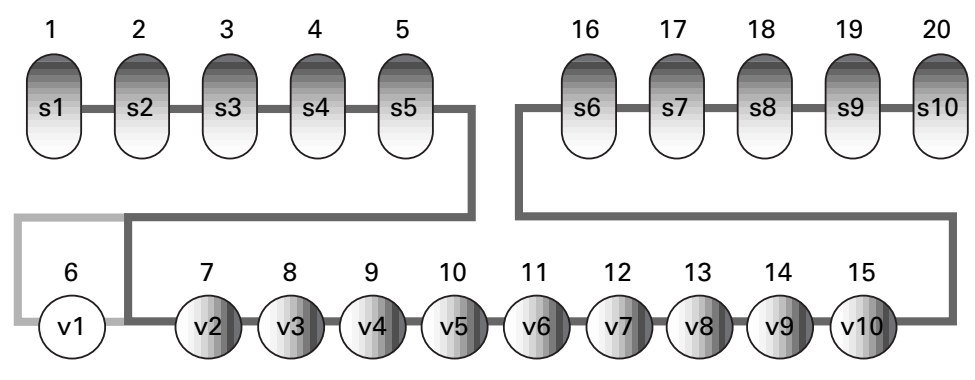

Variant exons

Figure 1 Schematic diagram of the structure of the CD44 gene. The standard exons (s1-10) encode the ubiquitously expressed standard protein isoform, CD44s. Combinations of the variant exons (v1-10) can be alternatively spliced between s5 and s6 to encode variant protein isoforms, CD44v. Unlike the mouse gene, exon 6 (v1) of the human gene contains a stop codon and is not normally included in human CD44 mRNA.
The families of proteins known collectively as cell adhesion molecules are cell surface glycoproteins that have large extracellular domains, a membrane spanning region, and an intracellular, cytoplasmic functional domain. These molecules are termed adhesion molecules because of their relatively strong bonding to specific ligands. However, the interaction is more complex than mere cell attachment, and may be more involved with the cell "sensing" the extracellular environment and sending out information to adjacent cells. Although all cell adhesion proteins are involved in cell-cell or cell-matrix interactions, they have also been shown to be involved in cell motility and migration, differentiation, cell signalling, and gene transcription. The expression patterns of cell adhesion molecules are being increasingly implicated in disease processes and are candidates for use in diagnostic pathology. For example, the organisation and function of epithelial cells is maintained by interaction with the underlying substrate and one of the characteristics of malignancy is the escape from the constraints imposed by the basement membrane.

The CD44 glycoproteins are well characterised members of the hyaluronate receptor family of cell adhesion molecules. This group is defined functionally, rather than structurally, and binds to ligands of the extracellular matrix (ECM). The major ligand is hyaluronate, which is an abundant extracellular polysaccharide found in mammalian ECM, but CD44 appears to have many varied functions dependant on the extracellular structure of the protein, which can be produced in a myriad of isoforms. The wide range of functional proteins is produced from a single gene by both alternative splicing and post-translational modification.

\section{CD44 structure}

GENE

The human CD44 gene has been mapped to the chromosomal locus $11 \mathrm{p} 13$ and is composed of two groups of exons (fig 1). ${ }^{1}$ One group, comprising exons $1-5$ and 16-20, are spliced together to form a transcript that encodes the ubiquitously expressed standard isoform (abbreviated to CD44s). The 10 variable exons 6-15 (also known as v1-10) can be alternatively spliced and included within the standard exons at an insertion site between exons 5 and $16 .^{2-5}$ Molecules containing the variable exons or their peptide products are designated CD44v and, in theory, alternative splicing would allow more than 1000 different CD44 variants to be generated. Further complexity of expression can be achieved by the alternative splicing of some of the standard 


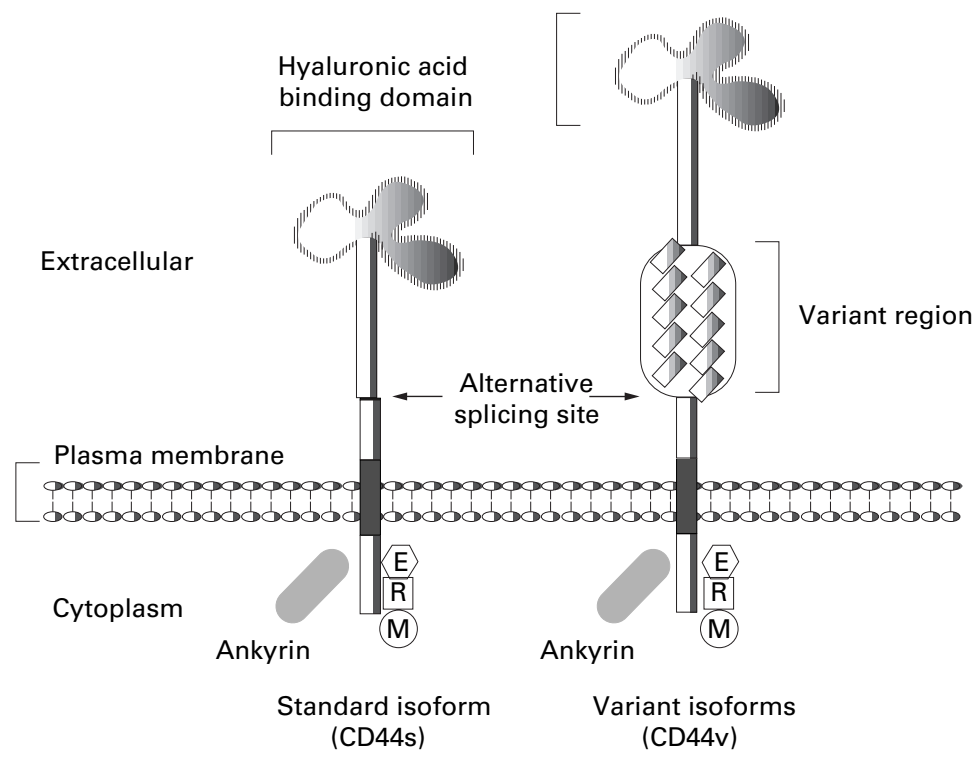

Figure 2 CD44 protein structure. The standard isoform binds its principal ligand, hyaluronic acid at the $\mathrm{N}$-terminal, distal extracellular domain. The inclusion of combinations of the variant exons (v1-10) within the extracellular domain can alter the binding affinity for hyaluronic acid and confer interaction with alternative ligands. The molecule interacts with the cytoskeleton through the binding of ankyrin and the ERM family (ezrin, radixin, moesin) to the cytoplasmic domain.

exons and by the use of cryptic splice sites. ${ }^{5}$ In the human gene, exon 6 (v1) contains a stop codon at the 17th amino acid and so, unlike in mice, this exon is not normally included in processed transcripts. $^{7}$

PROTEIN

The most abundant standard isoform of human CD44 protein (CD44s) contains 363 amino acids and has a theoretical molecular mass of $37 \mathrm{kDa}$. The protein consists of three regions, a 72 amino acid (aa) C-terminal cytoplasmic domain, a 21 aa transmembrane domain, and a 270 aa extracellular domain (fig 2 ). The cytoplasmic region is encoded by part of exon 18 and by exons 19 and 20. This highly conserved cytoplasmic tail can exist as a short or a more prevalent long form by the inclusion of the C-terminal exon. ${ }^{8}$ The hydrophobic transmembrane domain is encoded by exon 18 and is $100 \%$ conserved between mammalian species. The extracellular domain can be subdivided further into conserved and nonconserved regions. The N-terminal ectodomain, encoded by exons 1 to 5 is highly conserved $(\sim 85 \%)$ between mammalian species and is thought to fold into a globular tertiary structure by the formation of disulphide bonds between three pairs of cysteine residues. Also present in this section is a 100 aa region of homology with other hyaluronic acid (HA) binding proteins. This is termed the "link module" or CLP domain because of its resemblance to the HA binding domain of cartilage link protein. ${ }^{9}$ The variable region is the point at which up to 381 aa encoded by the 10 alternatively spliced variant exons are inserted at a site between exons 5 and 16 of the RNA transcript, corresponding to amino acid position 223 . The membrane proximal region of the extracellular domain, encoded by exons 16 and 17 is less conserved (35\%) between mammalian species and includes several carbohydrate modification sites.

POST-TRANSLATIONAL MODIFICATIONS

The apparent molecular mass of the CD44s protein, as estimated by gel electrophoresis, is $\sim 80 \mathrm{kDa}$ and the largest possible protein, containing peptides from all variant exons (referred to as "epican") can be over $200 \mathrm{kDa}$. This is far greater than the expected value as calculated from aa residues and is the result of the extensive post-translational modification of CD44 isoforms. There are at least five conserved consensus $\mathrm{N}$-glycosylation sites in the N-terminal ectodomain and two chondroitin sulphate attachment sites on the exon 5 product. Several potential $O$-linked glycosylation sites reside in the membrane proximal extracellular region and there are also consensus attachment sites for heparan sulphate, keratin sulphate, and sialic acid residues on the standard extracellular region. The alternatively spliced, variable exon products also have extensive potential modification sites, including serine/threonine rich regions for $O$-glycosylation, ${ }^{10}$ glycosaminoglycan attachment consensus SGXG motifs, ${ }^{11}$ and tyrosine sulphation. ${ }^{12}$

The degree of glycosylation can affect the ligand binding characteristics of the protein and therefore alter its function. Thus, the regulation of the amount and the type of posttranslational modification can add further diversity to the range of potential functions of CD44 isoforms.

\section{CD44 ligand binding}

HYALURONIC ACID

The principal ligand of CD44 is HA, a ubiquitous component of the ECM. HA is a linear, polymeric glycosaminoglycan and there are at least three sites for its binding on the CD44 molecule: one in the "link" domain encoded by exon $2,{ }^{13}$ and the other two overlap in the region encoded by exon $5 .{ }^{14}$ The HA binding sites consist of basic aa clusters, with specific arginine residues being essentially required, as shown by mutation studies. ${ }^{15} 16$ However, although all CD44 isoforms contain the HA recognition site, not all cells expressing CD44 bind the HA ligand constitutively. Cells can express CD44 in an active, an inducible, or an inactive state with respect to HA binding. The differences in the HA binding state of CD44 are cell specific and have been shown to be related to post-translational modification patterns. Inhibition of $\mathrm{N}$-glycosylation enhances HA binding, ${ }^{17}$ and abrogation of glycosylation by mutation at specific sites can convert the inducible form to the constitutively active form. In certain cell types, inactive CD44 surface protein can be rapidly induced to bind HA by interaction with specific antibodies. The induced binding state can also be achieved more slowly by incubation of the cells with stimulating agents such as phorbol esters or interleukins. ${ }^{18}$ Detailed mutational analysis of basic a clusters in the cytoplasmic domain has defined specific arginine and lysine residues 
through which protein kinase C (PKC) stimulating reagents differentially regulate the binding of CD44 to hyaluronic acid. ${ }^{19}$

\section{OSTEOPONTIN}

There are reports of interactions between CD44 and another member of the ECM, osteopontin. Co-localised immunoreaction of osteopontin and CD44v in tumour cells concomitant with cluster formation in the lymphatic vessels has been observed, suggesting that interaction between osteopontin and CD44 may parallel lymphogenous metastasis. ${ }^{20}$ Weber et al have reported that osteopontin binds to CD44 in a specific, dose dependent manner and that the binding is sensitive to competition by both hyaluronate and anti-CD44 antibodies. ${ }^{21}$ Osteopontin is a cytokine secreted by several cell types and it induces cellular chemotaxis. It has been suggested that the coupling of osteopontin secretion and the expression of CD44 could cause migration and metastasis of tumour cells to specific sites. ${ }^{22}$

\section{OTHER EXTRACELLULAR LIGANDS}

The attachment of the polysaccharide chondroitin to sites on the CD44 protein (exon 5) can lead to a number of indirect extracellular matrix binding interactions. Collagen, ${ }^{23}{ }^{24}$ fibronectin, and laminin can bind to CD44 in this way. ${ }^{25}$ Serglycin, a family of proteoglycans named for its Ser-Gly dipeptide repeats, has also been reported to bind to CD44 indirectly. ${ }^{26}$ Chondroitin sulphate modified serglycin is secreted from haemopoietic cells and its interaction with CD44 leads to the aggregation, adherence, and activation of lymphoid cells. ${ }^{27}{ }^{28} \mathrm{CD} 44$ isoforms containing the alternatively spliced exon v3 can be modified with heparan sulphate at a Ser-Gly-X-Gly motif and are then able to bind basic fibroblast growth factor (b-FGF) and heparin binding epidermal growth factor (HB-EGF). ${ }^{29}{ }^{11}$ The manipulation of glycosylation patterns by the alternative splicing of CD44 is an important mechanism for the achievement of multifunctional products from a single gene. ${ }^{10}{ }^{30}$

\section{Interaction of CD44 with cellular proteins} MULTIMERISATION

In common with other cell surface molecules, there is evidence that ligand binding may be enhanced by the multimerisation of CD44. The effect of antibodies that induce CD44-HA binding requires multivalency, suggesting that CD44 clustering is required for such induced binding. ${ }^{31}$ Sleeman et al demonstrated that reduction sensitive dimerisation of CD $44 \mathrm{v}$ can occur, and that glycosylation inhibition that abrogates CD44v-HA binding also prevents oligomerisation. ${ }^{32}$ Furthermore, although CD44 that lacks the cytoplasmic domain does not bind HA efficiently, ${ }^{33}$ synthetic CD44 molecules that can form disulphide bonded dimers retain HA binding, even when the cytoplasmic domain of the CD44 dimer is absent. ${ }^{34}$

HER2

It is now apparent that CD44 interacts with the HER2 (p185, c-ErbB2) cell surface protein, a tyrosine kinase and a member of the EGF receptor family. Overexpression of HER2 by tumour cells is associated with a poor prognosis in many studies of breast and ovarian cancer. The HER2 receptor can bind the ligand heregulin and incubation of $\mathrm{SKBr} 3$ breast cancer cells with heregulin increases the adhesion of these cells to plastic and the invasiveness of the tumour cells into Matrigel membranes. ${ }^{35}$ Interestingly, this heregulin treatment also increased expression of the CD44 adhesion molecule and invasion of Matrigel membranes was partially blocked by anti-CD44 antibodies, indicating a dual role for these adhesion molecules in the invasion process. Bourguignon et al examined the interaction between CD44s and the her2 protooncogene product in an ovarian carcinoma cell line. Anti-CD44 mediated immunoprecipitation indicated that both CD44s and HER2 are found on the cell surface and that these two molecules are physically linked to each other via interchain disulphide bonds. It was also shown that HA binding stimulates HER2 tyrosine kinase activity, leading to an increase in ovarian carcinoma cell growth. ${ }^{36}$ Furthermore, they found that CD44s expression and CD44s mediated cell adhesion to HA were both significantly reduced after transfection of the cell line with the adenovirus $5 \mathrm{E} 1 \mathrm{~A}$ gene, which is known to repress the synthesis of HER2. Although studies using in vitro analysis of fusion proteins have shown that HA binding is largely dependent on the structure of the CD44 molecule alone, ${ }^{16}$ data suggest that the expression and function of the CD44 and HER2 cell surface molecules are coupled to some extent.

Immunochemical analysis of melanoma cell lines has shown that CD44 co-localises and co-precipitates with the proteoglycan surface molecule NG2. ${ }^{37} \mathrm{NG} 2$ is also associated with $\alpha 4 \beta 1$ integrin and, as with HER2, this association with other cell surface molecules suggests that CD44 may be only one part of large, multifunctional complexes, in which a balance of complementary or competitive ligand binding exists.

\section{Interaction with intracellular proteins}

The status of the cytoplasmic domain of CD44 can influence the binding of extracellular HA (see above), presumably by membrane localisation, involving clustering or dimerisation. However, the main role for the cytoplasmic domain is to transduce signals from extracellular stimuli. ${ }^{38}$ The cytoplasmic domain has been shown to bind to a number of intracellular proteins directly. An ankyrin binding domain has been identified ${ }^{39}{ }^{40}$ and binding appears to be enhanced by palmitoylation of CD44 amino acid residues. ${ }^{41}$ In haemopoietic cells, it has been shown that protein 4.1 can bind directly to CD44 and this interaction prevents subsequent ankyrin binding. ${ }^{42} \mathrm{~A}$ correlation between the ability to bind ankyrin and HA has been proposed $^{4043}$ and, although it is not demonstrable in all cell types, ${ }^{34}$ it is clear that dynamic intracellular and extracellular interactions can be facilitated by CD44 proteins. As an example 
of this, it has been reported that a CD44v3,8-10 isoform, present in a metastatic breast cell line, can interact with ankyrin via the cytoplasmic domain and is found closely associated with an active matrix metalloproteinase in a complex within pseudopodia structures during cell migration. ${ }^{44}$

CD44 interactions with the ERM family (ezrin, radixin, and moesin) of proteins have also been described. CD44 has been shown to co-precipitate and co-localise with ERM proteins, ${ }^{45}$ and a region of basic amino acids in the cytoplasmic tail has been identified as essential for the binding of moesin ${ }^{46}$ and ezrin. ${ }^{47}$ Ankyrin and ERM proteins connect elements of the plasma membrane with the actin filament network of the cell and thus a direct link between CD44 and intracellular scaffold structures can be envisioned, facilitating cell motility and migration, as well as membrane localisation of CD $44 .^{46}$

\section{CD44 expression}

Most mammalian cells predominantly express the CD44s isoform, but some epithelial cells also express a larger isoform (CD44E), which has the products of exons v8-10 included (fig 1), often in excess of CD $44 \mathrm{~s} .{ }^{48}$ The expression of CD44 isoforms containing combinations of the other variant exons is far more restricted in normal tissues. However, the expression of variant isoforms is detectable in haemopoietic cells, ${ }^{49}$ particularly in peripheral blood mononuclear cells ${ }^{50}$ and in reactive lymph node cells. $^{49} 51$ The expression of v6 containing isoforms has been observed in the ducts of breast ${ }^{52}$ and pancreas, ${ }^{53}{ }^{54}$ and $\mathrm{v} 4$ isoforms may be expressed in normal urothelium. ${ }^{55} \mathrm{~A}$ few cell types do not express any CD44 isoforms, including hepatocytes, pancreatic acinar cells, and cells of the tubules of the kidney and pancreas. ${ }^{52} 56$

\section{CD44 function}

The multiple functions of the CD44 family of proteins are centered around the binding of $\mathrm{HA}$ and, to a lesser extent, other extracellular molecules. Functional diversity is achieved by the alternative splicing of the pre-mRNA, and the fine tuning of ligand binding can be achieved by post-translational modification and dynamic interaction with other cellular factors, as discussed above. The balance of interactions between cell surface molecules and between those molecules and the ECM will determine the positional address and the migrational status of specific cell types.

CELL ADHESION

The CD44s protein is involved primarily in the maintenance of three dimensional organ/tissue structure. Epithelia undergoing proliferation and cells under repair appear to upregulate both CD44 and HA production enabling the production of, and then attachment to, a structural scaffold during expansion. ${ }^{57}{ }^{58} \mathrm{HA}$ accumulates in angiogenesis, ${ }^{59}$ wound healing, ${ }^{58}$ and embryonic cell migration, ${ }^{6061}$ and because migration of cells across an HA substrate has been described, ${ }^{33} \mathrm{HA}$ appears to serve as a ligand support for CD44 mediated cell movement.

CD44 molecules can also mediate the aggregation of cells. This can be achieved via multivalent $\mathrm{HA}$ binding by CD44 on adjacent cells or via inter-CD44 binding via attached glycosylation moieties. ${ }^{62} \mathrm{HA}$ dependant binding can cause aggregation of macrophages, lymphocytes, and fibroblasts..$^{63}$

The binding of HA can also induce gene expression. A number of inflammatory genes that can be induced in macrophages by HA oligomers have been identified. These include several members of the chemokine gene family, and the inducible form of nitric oxide synthase. HA fragments as small as hexamers are capable of inducing these effects and monoclonal antibodies to the HA receptor CD44 inhibits HA induced gene expression. ${ }^{65}$

ROLE IN LYMPHOCYTE FUNCTION

Several lymphocyte functions appear to be dependant upon CD44 expression. Increased surface levels of CD44 proteins are characteristic of $T$ cell activation after encounter with its cognate antigen. ${ }^{66}$ Cell surface CD44 on lymphocytes can mediate the adhesion of lymphocytes to vascular endothelial cells via binding of HA, and this interaction is used for activated $\mathrm{T}$ cell extravasation into sites of inflammation in mice ${ }^{67}$ and in humans. ${ }^{68}$ This targeting of lymphocytes to effector sites by CD44-HA binding is enhanced by the induction of HA synthesis in vascular endothelium by the proinflammatory cytokines, tumour necrosis factor $\alpha(\mathrm{TNF}-\alpha)$ and interleukin $1 \beta$ (IL-1 $\beta) .{ }^{69}$ Accordingly, the presence of CD44 splice variants appears to be obligatory for the migration and function of Langerhans cells and dendritic cells from peripheral organs to lymph nodes for antigen presentation. ${ }^{70}$

\section{CD44 in neoplasia}

The expression of multiple CD44 isoforms and the resulting HA binding profile can influence tumour growth and development. Initial studies showed that tumour tissues contained a number of unusual CD44 transcripts relative to those present in corresponding normal tissues. $^{71} 72$ In addition, tumour cell lines with raised concentrations of CD44 proteins were shown to be capable of forming more aggressive tumours in animal experiments. ${ }^{73} 74$ Changes in the pattern of expression of a cell adhesion molecule in a tissue are likely to disrupt normal epithelial-mesenchymal interactions and thus contribute to structural and functional disorganisation, a characteristic of cancer.

The requirement for CD44-HA binding in tumour development has been shown by the suppression of tumour formation in the presence of soluble human CD44s fusion proteins. ${ }^{75}{ }^{76}$ Administration of a mutant CD 44 fusion protein, which was incapable of binding HA, did not abrogate tumour development. ${ }^{77}$ Local injection of hyaluronan oligomers can also inhibit tumour formation by melanoma 
cells, ${ }^{78}$ presumably also by interfering with endogenous CD44-HA binding.

Interestingly, this type of competitive inhibition can induce apoptosis. Expression of transfected soluble CD44 isoforms was shown to suppress murine mammary carcinoma cells from developing tumours in vivo and this was found to be the result of large scale apoptosis. ${ }^{79}$ Furthermore, detachment from the substratum of cultured fibroblasts by anti-CD44 antibodies can also lead to apoptosis. ${ }^{80}$ Conversely, there is evidence that CD44 engagement by HA can protect lymphocytes from glucocorticoid mediated apoptosis ${ }^{81}$ and colon tumour cells from anti-integrin antibody induced apoptosis. $^{82}$

ROLE IN METASTASIS

Much interest and investigative effort has centred on the possible involvement of CD44 isoforms in tumour metastasis. This is partly because of the many parallels between lymphocyte migration and the process of metastasis. In contrast to the ubiquitous expression of CD44s in most cell types, activated lymphocytes express multiple CD44 variant isoforms. ${ }^{518384}$ Gunthert et al first investigated CD44 function in metastasis directly by transfecting plasmids expressing CD44s or CD44v isoforms into non-metastatic, rat pancreatic carcinoma cells. ${ }^{85}$ Expression of the CD $44 \mathrm{~s}$ gene had no effect in this tumour but CD44v expression conveyed metastatic competence to these cells in an athymic mouse model. Expression of constructs containing variant exon v6 and v7 sequences could establish metastatic potential. ${ }^{85} 86$ The ability of the CD44v expressing cells to metastasise was not dependant on HA binding because a CD44 exon v6 specific antibody that blocked metastasis formation in this system ${ }^{87}$ did not interfere with HA binding. ${ }^{88}$ Two other groups subsequently provided evidence of a correlation between raised CD44 expression and metastatic capability in cultured human melanoma ${ }^{73}$ and lymphoma cell lines. ${ }^{74}$ However, in these reports it was $\mathrm{CD} 44 \mathrm{~s}$ rather than $\mathrm{CD} 44 \mathrm{v}$ isoforms that were associated with metastatic behaviour. More recent studies have investigated the role of CD44 in metastasis in other ways. In one study, a metastatic murine mammary carcinoma transfected to secrete soluble isoforms of CD44 lost the ability to bind and internalise hyaluronan and developed few or no tumours. ${ }^{76}$ Reeder et al used an antisense mRNA approach to downregulate CD44 variant isoforms containing CD44v6 in the metastatic colorectal tumour cell line HT29. Expression of antisense exon 10 (v6) transcripts had no effect on HT29 tumour cell proliferation in vitro or on the ability of the cells to bind immobilised hyaluronan, but it did result in a reduced capacity to form liver metastases in nude mice. ${ }^{89}$ In line with the analogy of lymphocyte migration as a model of metastasis, a monoclonal antibody directed against a CD44 exon v6 epitope blocked both metastasis formation and lymphocyte activation, and caused accelerated immune re- sponses in transgenic mice engineered to express CD $44 v 4-7$ on T cells. ${ }^{90}$

These observations suggest that cell surface CD44 function promotes tumour cell survival in invaded tissue. However, the question of a role for CD44 in metastasis remains open because there is evidence that CD44 alone is not responsible for metastatic capability. A murine lymphoma cell line with both alleles of the CD44 gene removed was shown to retain its invasiveness and metastatic potential in vivo, despite losing all hyaluronan binding ability. ${ }^{91}$ CD $44 \mathrm{v}$ does appear to be overexpressed in some metastatic lineages, but not always so. ${ }^{92}$ It is also possible that a pattern of expression that is advantageous for tumour cell migration may be tissue specific. Zahalka et al showed that the presence of an anti-CD44 antibody or hyaluronidase prevented lymph node infiltration but had no effect on spleen invasion by murine lymphoma cells. ${ }^{93}$

DIAGNOSTIC APPLICATIONS

The use of CD44 analysis as a diagnostic and/or prognostic marker was first suggested when it was shown that CD44 expression was greatly altered in human tumour tissues comp ared with the tissue of origin. ${ }^{94}$ Since that time, there have been very many studies of CD44 expression in human tissue samples and, although there are trends in patterns of expression, a truly tumour specific isoform, or pattern of expression, has yet to be defined..$^{95}$ We and others have performed many studies that have analysed CD44 protein concentrations ${ }^{96} 97$ or mRNA expression in solid tumour tissue extracts $^{98-100}$ but, in the absence of tumour specific isoforms, such analysis is not yet reliable enough to challenge pathological diagnosis on morphological parameters. The most promising area for the use of CD44 in diagnosis is in the analysis of body fluids. We have achieved high levels of accuracy in the detection of bladder cancer by the analysis of non-invasively obtained urine samples ${ }^{101-103}$ and, in this area, molecular diagnosis can be as reliable as conventional cytology.

Recently, studies have considered the analysis of soluble CD44 in body fluids for diagnosis. The soluble CD44 isoforms found in the serum lack a cytoplasmic tail and do not appear to be associated with other proteins or with the ligand hyaluronan. ${ }^{104}$ There does appear to be a correlation between soluble CD44 concentrations and tumour burden in patients with lymphomas and these values return to within normal ranges if the patient responds to treatment. ${ }^{105} 106$ The mechanism by which tumours produce more soluble CD44 is unknown but it could result from the misprocessing of transcripts, including intron retention or exon skipping. Splicing errors or inclusion of stop codons would lead to the translation of truncated proteins that may lack the transmembrane region and would therefore be secreted.

ABERRANT CD44 TRANSCRIPT PROCESSING Analysis of tumour cell CD44 mRNA consistently reveals an extensive array of alternatively 
spliced transcripts, including many that are larger than would be predicted from the known structure of the gene. Further analysis has revealed that the level of misprocessing of CD44 transcripts is greatly increased in tumour cells. Examples of this are intron retention and the use of cryptic splice sites.

Compromised CD44 intron removal was first observed in exfoliated urothelia of bladder cancer patients, ${ }^{102}$ and was subsequently found also to occur in breast carcinoma tissues ${ }^{107}$ and in colon carcinoma, ${ }^{103}$ and can involve introns of the variant and standard regions of the gene. ${ }^{108}$ Further evidence of tumour cell CD44 misprocessing has been reported, revealing that tumour cells produce CD44 transcripts that have been aberrantly processed using "cryptic" splice sites. ${ }^{6}$ As in the case of intron retention, these events can lead to the translation of truncated, non-functional peptides. The understanding of the processing of CD44 pre-mRNA is an area of great interest and the mechanisms of CD44 splicing are beginning to be elucidated. ${ }^{109110}$

\section{PLASTICITY OF EXPRESSION}

Tumour cell expression of CD44 isoforms does not remain static during changing growth conditions in vitro ${ }^{111}$ or in vivo. ${ }^{112}$ The transfection of the human CD44s gene into mouse 3T3 cells revealed remarkable plasticity of expression during tumour progression and metastasis in a nude mouse model. ${ }^{113} 114$ Expression of CD44s induced tumorigenesis, HA binding, and micrometastatic competence to multiple organs. However, large primary tumours and overt metastases lost CD44s expression and HA binding, and this was shown to be the result of hypermethylation of the transfected human CD 44 s gene. These studies indicate that CD44s expression and the binding of CD44s to HA is important for tumorigenesis and metastatic competence, but that downregulated expression is beneficial to advanced tumour growth.

A large body of evidence supports the view that altered CD44 expression correlates with tumour growth and metastasis. However, there appears to be considerable cell-type and tumour-type specificity for the functions of this family of molecules. Whether this molecule can become the focus of clinical intervention remains to be seen, but it is a candidate marker that will probably be included in multiplex molecular assays in the future.

\section{Summary}

The CD44 family of transmembrane proteins is perhaps the best example yet studied of the way an organism can achieve multiple and diverse functions from a single gene. Detailed functional studies are revealing increasingly complex roles for the CD44 family of proteins in physiological and pathological processes. The multifunctional aspect of CD44 cell adhesion molecules is achieved by the balance of a number of variable parameters that the cell uses in the expression of this gene. These include the amount of total CD44, alternative splicing choices, the balance of many types of post-translational modifications, and the active/inactive HA binding state of the cell surface protein. Furthermore, the homo/hetero multimerisation of the protein, the interaction with other adhesion molecules (which can compete for ligand binding), and the amount of soluble CD44 protein in the extracellular space also need to be considered. Further characterisation of the function of CD $44 \mathrm{v}$ proteins and how they interact with other cellular components and with the extracellular environment is necessary before a comprehensive understanding of the physiological roles of the CD44 cell adhesion molecules can be achieved.

1 Goodfellow PN, Banting G, Wiles MV, et al. The gene, MIC4, which controls expression of the antigen defined by monoclonal antibody F10.44.2, is on human chromosome 1. Eur F Immunol 1982;12:659-63.

2 Hofmann M, Rudy W, Zoller M, et al. CD44 splice variants confer metastatic behavior in rats: homologous sequences are expressed in human tumor cell lines. Cancer Res 1991; 51:5292-7.

3 Dougherty GJ, Landorp PM, Cooper DL, et al. Molecular cloning of CD44R1 and CD44R2, two novel isoforms of the human CD44 lymphocyte "homing" receptor expressed by hemopoietic cells. ₹ Exp Med 1991;174:1-5.

4 Tolg C, Hofmann M, Herrlich P, et al. Splicing choice from ten variant exons establishes CD44 variability. Nucleic Acids Res 1993;21:1225-9.

5 Screaton GR, Bell MV, Jackson DG, et al. Genomic structure of DNA encoding the lymphocyte homing recep-
tor CD44 reveals at least 12 alternatively spliced exons. tor CD44 reveals at least 12 alternatively
Proc Natl Acad Sci USA 1992;89:12160-4

6 Ermak G, Jennings T, Boguniewicz A, et al. Novel CD44 messenger RNA isoforms in human thyroid and breast tissues feature unusual sequence rearrangements. Clin Cancer Res 1996;2:1251-4.

7 Screaton GR, Bell MV, Bell JI, et al. The identification of a new alternative exon with highly restricted tissue expres-
sion in transcripts encoding the mouse Pgp-1 (CD44) homing receptor. Comparison of all 10 variable exons between mouse, human, and rat. F Biol Chem 1993;268: 12235-8.

8 Goldstein LA, Butcher EC. Identification of mRNA that encodes an alternative form of H-CAM (CD44) in lymphoid and nonlymphoid tissues. Immunogenetics 1990; 32:389-97.

9 Kohda D, Morton CJ, Parkar AA, et al. Solution structure of the link module: a hyaluronan-binding domain involved in extracellular matrix stability and cell migration. Cell 1996; 86:767-75.

10 Bennett KL, Modrell B, Greenfield B, et al. Regulation of $\mathrm{CD} 44$ binding to hyaluronan by glycosylation of variably spliced exons. F Cell Biol 1995;131:1623-33.

11 Jackson DG, Bell JI, Dickinson R, et al. Proteoglycan forms of the lymphocyte homing receptor CD44 are alternatively spliced variants containing the v3 exon. 7 Cell Biol 1995;128:673-85.

12 Sleeman JP, Rahmsdorf U, Steffen A, et al. CD44 variant exon 55 encodes a tyrosine that is sulphated. Eur $\mathcal{F}$ Biochem 1998;255:74-80.

13 Yang B, Yang BL, Savani RC, et al. Identification of a common hyaluronan binding motif in the hyaluronan binding proteins RHAMM, CD44 and link protein. EMBO f 1994; 13:286-96.

14 Liao HX, Lee DM, Levesque MC, et al. N-terminal and central regions of the human CD44 extracellular domain participate in cell surface hyaluronan binding. F Immunol 1995;155:3938-45.

15 Bajorath J, Greenfield B, Munro SB, et al. Identification of CD44 residues important for hyaluronan binding and delineation of the binding site. 7 Biol Chem 1998;273:33843.

16 Peach RJ, Hollenbaugh D, Stamenkovic I, et al. Identification of hyaluronic acid binding sites in the extracellular domain of CD 44. F Cell Biol 1993;122:257-64.

17 Lesley J, English N, Perschl A, et al. Variant cell lines

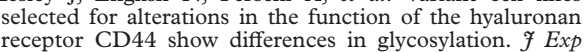
receptor CD44 show
Med 1995;182:431-7.

18 Lesley J, Hyman R, Kincade PW. CD44 and its interaction with extracellular matrix. Adv Immunol 1993;54:271-335.

9 Liu D, Liu T, Sy MS. Identification of two regions in the cytoplasmic domain of CD44 through which PMA, calcium, and foskolin differentially regulate the binding of CD44 to hyaluronic acid [in process citation]. Cell Immunol 1998;190:132-40.

20 Ue T, Yokozaki H, Kitadai Y, et al. Co-expression of osteopontin and CD44v9 in gastric cancer. Int $\mathcal{F}$ Cancer 1998;79:127-32.

21 Weber GF, Ashkar S, Cantor H. Interaction between CD44 and osteopontin as a potential basis for metastasis formation. Proc Assoc Am Physicians 1997;109:1-9. 
22 Weber GF, Ashkar S, Glimcher MJ, et al. Receptor-ligand interaction between CD44 and osteopontin (Eta-1). Science 1996;271:509-12.

23 Faassen AE, Schrager JA, Klein DJ, et al. A cell surface chondroitin sulfate proteoglycan, immunologically related to $\mathrm{CD} 44$, is involved in type I collagen-mediated melanoma cell motility and invasion. $\mathcal{F}$ Cell Biol 1992;116 521-31.

24 Knutson JR, Iida J, Fields GB, et al. CD44/chondroitin sulfate proteoglycan and alpha 2 beta 1 integrin mediate human melanoma cell migration on type IV collagen and invasion of basement membranes. Mol Biol Cell 1996;7: invasion

25 Jalkanen S, Jalkanen M. Lymphocyte CD44 binds the $\mathrm{COOH}$-terminal heparin-binding domain of fibronectin. $\mathcal{f}$ Cell Biol 1992;116:817-25.

26 Toyama-Sorimachi N, Miyasaka M. A novel ligand for CD44 is sulfated proteoglycan. Int Immunol 1994;6:65560.

27 Toyama-Sorimachi N, Sorimachi H, Tobita Y, et al. A novel ligand for CD44 is serglycin, a hematopoietic cell lineagespecific proteoglycan. Possible involvement in lymphoid cell adherence and activation. F Biol Chem 1995;270:743744.

28 Toyama-Sorimachi N, Kitamura F, Habuchi H, et al. Widespread expression of chondroitin sulfate-type serglycins with CD44 binding ability in hematopoietic cells. $\mathcal{F}$ Bio Chem 1997;272:26714-19.

29 Bennett KL, Jackson DG, Simon JC, et al. CD44 isoforms containing exon V3 are responsible for the presentation of heparin-binding growth factor. F Cell Biol 1995;128:68798.

30 Piepkorn M, Hovingh P, Bennett KL, et al. Chondroitin sulphate composition and structure in alternatively spliced
CD44 fusion proteins. Biochem $\mathcal{F}$ 1997;327:499-506.

31 Lesley J, Kincade PW, Hyman R. Antibody-induced activation of the hyaluronan receptor function of CD44 requires multivalent binding by antibody. Eur f Immunol 1993;23: 1902-9.

32 Sleeman J, Rudy W, Hofmann M, et al. Regulated clustering of variant CD44 proteins increases their hyaluronate binding capacity. F Cell Biol 1996;135:1139-50.

33 Thomas L, Byers HR, Vink J, et al. CD44H regulates tumor cell migration on hyaluronate-coated substrate. $\mathcal{F}$ Cell Biol 1992;118:971-7.

34 Perschl A, Lesley J, English N, et al. Role of CD44 cytoplasmic domain in hyaluronan binding. Eur $\mathcal{f}$ Immunol mic domain in hya

35 Xu FJ, Stack S, Boyer C, et al. Heregulin and agonistic antip185(c-erbB2) antibodies inhibit proliferation but increase invasiveness of breast cancer cells that overexpress p185 (c-erbB2): increased invasiveness may contribute to poo prognosis. Clin Cancer Res 1997;3:1629-34.

36 Bourguignon LY, Zhu $\mathrm{H}$, Chu A, et al. Interaction between the adhesion receptor, $\mathrm{CD} 44$, and the oncogene product, p185HER2, promotes human ovarian tumor cell activation. F Biol Chem 1997;272:27913-18.

37 Burg MA, Grako KA, Stallcup WB. Expression of the NG2 proteoglycan enhances the growth and metastatic properproteoglycan enhances the growth and metastatic proper-
ties of melanoma cells. $\mathcal{F}$ Cell Physiol 1998;177:299-312.

38 Bourguignon LYW, Zhu D, Zhu H. CD44 isoformcytoskeleton interaction in oncogenic signaling and tumor progression [in process citation]. Front Biosci 1998;3 D637-49.

39 Lokeshwar VB, Fregien N, Bourguignon LY. Ankyrinbinding domain of CD44 (GP85) is required for the expression of hyaluronic acid-mediated adhesion function. f Cell Biol 1994;126:1099-109.

40 Zhu D, Bourguignon LY. The ankyrin-binding domain of CD44s is involved in regulating hyaluronic acid-mediated functions and prostate tumor cell transformation. Cell Moti Cytoskeleton 1998;39:209-22.

41 Bourguignon LY, Kalomiris EL, Lokeshwar VB. Acylation of the lymphoma transmembrane glycoprotein, GP85, may be required for GP85-ankyrin interaction. F Biol Chem 1991;266:11761-5.

42 Nunomura W, Takakuwa Y, Tokimitsu R, et al. Regulation of CD44-protein 4.1 interaction by $\mathrm{Ca}^{2+}$ and calmodulin Implications for modulation of CD44-ankyrin interaction. f Biol Chem 1997;272:30322-8.

43 Liu D, Sy MS. A cysteine residue located in the transmembrane domain of CD44 is important in binding of CD44 to hyaluronic acid. $\mathcal{F}$ Exp Med 1996;183:1987-94.

44 Bourguignon LY, Gunja-Smith Z, Iida N, et al. CD44v $(3,8-10)$ is involved in cytoskeleton-mediated tumor cell migration and matrix metalloproteinase (MMP-9) associmigration and matrix metalloproteinase (MMP-9) associ-
ation in metastatic breast cancer cells. $\mathcal{F}$ Cell Physio ation in metasta

45 Tsukita S, Oishi K, Sato N, et al. ERM family members as molecular linkers between the cell surface glycoprotein CD44 and actin-based cytoskeletons. F Cell Biol 1994;126: 391-401.

46 Yonemura S, Hirao M, Doi Y, et al. Ezrin/radixin/moesin (ERM) proteins bind to a positively charged amino acid cluster in the juxta-membrane cytoplasmic domain of

7 Legg JW, Isacke CM. Identification and functional analysis of the ezrin-binding site in the hyaluronan receptor, CD44. Curr Biol 1998;8:705-8.

48 Iida N, Bourguignon LY. New CD44 splice variants associated with human breast cancers. F Cell Physiol 1995;162: $127-33$.
49 Stauder R, Eisterer W, Thaler J, et al. CD44 variant isoforms in non-Hodgkin's lymphoma: a new independent prognostic factor. Blood 1995;85:2885-99.

50 Salles G, Zain M, Jiang WM, et al. Alternatively spliced CD44 transcripts in diffuse large-cell lymphomas: characterization and comparison with normal activated B cells and epithelial malignancies. Blood 1993;82:3539-47.

51 Arch R, Wirth K, Hofmann M, et al. Participation in normal mmune responses of a metastasis-inducing splice variant of CD44 [see comments]. Science 1992;257:682-5.

52 Terpe HJ, Stark H, Prehm P, et al. CD44 variant isoforms are preferentially expressed in basal epithelial of nonmalignant human fetal and adult tissues. Histochemistry 1994;101:79-89.

53 Gansauge F, Gansauge S, Zobywalski A, et al. Differential expression of CD44 splice variants in human pancreatic adenocarcinoma and in normal pancreas. Cancer Res 1995; 55:5499-503.

54 Hong RL, Pu YS, Chu JS, et al. Correlation of expression of CD44 isoforms and E-cadherin with differentiation in CD44 isoforms and E-cadherin with differentiation in Cancer Lett 1995;89:81-7.

55 Southgate J, Trejdosiewicz LK, Smith B, et al. Patterns of splice variant CD44 expression by normal human urothelium in situ and in vitro and by bladder-carcinoma cell lines. Int f Cancer 1995;62:449-56.

56 Mackay CR, Terpe HJ, Stauder R, et al. Expression and modulation of CD44 variant isoforms in humans. $f$ Cell Biol 1994;124:71-82

57 Alho AM, Underhill CB. The hyaluronate receptor is preferentially expressed on proliferating epithelial cells. $\mathcal{f}$ Cell Biol 1989;108:1557-65.

58 Jain $\mathrm{M}, \mathrm{He} \mathrm{Q}$, Lee WS, et al. Role of CD44 in the reaction of vascular smooth muscle cells to arterial wall injury. $\mathcal{F}$ Clin Invest 1996;98:877.

59 Trochon V, Mabilat C, Bertrand P, et al. Evidence of involvement of CD44 in endothelial cell proliferation, migration and angiogenesis in vitro. Int $\mathcal{f}$ Cancer 1996;66: 664-8.

60 Sherman L, Sleeman J, Dall P, et al. The CD44 proteins in embryonic development and in cancer. Curr Top Microbiol Immunol 1996;213:249-69.

61 Wainwright D, Sherman L, Sleeman J, et al. A splice variant of CD44 expressed in the rat apical ectodermal ridge contributes to limb outgrowth. Ann NY Acad Sci 1996;785: 345-9.

62 Cooper DL, Dougherty GJ. To metastasize or not? Selection of CD44 splice sites. Nat Med 1995;1:635-7.

63 Green SJ, Tarone G, Underhill CB. Aggregation of macrophages and fibroblasts is inhibited by a monoclonal antibody to the hyaluronate receptor. Exp Cell Res 1988;178:224-32.

64 Underhill C, Dorfman A. The role of hyaluronic acid in intercellular adhesion of cultured mouse cells. Exp Cell Res 1978;117:155-64.

65 McKee CM, Penno MB, Cowman M, et al. Hyaluronan (HA) fragments induce chemokine gene expression in alveolar macrophages. The role of HA size and CD44. F Clin Invest 1996;98:2403-13.

66 DeGrendele HC, Kosfiszer M, Estess P, et al. CD44 activation and associated primary adhesion is inducible via $\mathrm{T}$ cell receptor stimulation. $\mathcal{F}$ Immunol 1997;159:2549-53.

67 DeGrendele HC, Estess P, Siegelman MH. Requirement for CD44 in activated $\mathrm{T}$ cell extravasation into an inflammatory site. Science 1997;278:672-5.

68 Estess P, DeGrendele HC, Pascual V, et al. Functional activation of lymphocyte CD44 in peripheral blood is a marker of autoimmune disease activity [see comments]. F Clin Invest 1998;102:1173-82.

69 Mohamadzadeh M, DeGrendele $\mathrm{H}$, Arizpe $\mathrm{H}$, et al. Proinflammatory stimuli regulate endothelial hyaluronan
expression and CD $44 / \mathrm{HA}$-dependent primary adhesion. $\mathcal{f}$ expression and CD 44/HA-depe

70 Weiss JM, Renkl AC, Sleeman J, et al. CD44 variant isoforms are essential for the function of epidermal Langerhans cells and dendritic cells [in process citation]. Cell Adhes Commun 1998;6:157-60.

71 Stamenkovic I, Amiot M, Pesando JM, et al. A lymphocyte molecule implicated in lymph node homing is a member of the cartilage link protein family. Cell 1989;56:1057-62.

72 Stamenkovic I, Aruffo A, Amiot M, et al. The hematopoietic and epithelial forms of CD44 are distinct polypeptides with different adhesion potentials for hyaluronate-bearing cells. different adhesion poten

73 Birch M, Mitchell S, Hart IR. Isolation and characterization of human melanoma cell variants expressing high and low levels of CD44. Cancer Res 1991;51:6660-7.

74 Sy MS, Guo YJ, Stamenkovic I. Distinct effects of two CD44 isoforms on tumor growth in vivo. $\mathcal{F}$ Exp Med 1991; 174:859-66.

75 Sy MS, Guo YJ, Stamenkovic I. Inhibition of tumor growth in vivo with a soluble CD44-immunoglobulin fusion protein. F Exp Med 1992;176:623-7.

76 Zawadzki V, Perschl A, Rosel M, et al. Blockade of metastasis formation by CD44-receptor globulin. Int 7 Cancer 1998;75:919-24.

77 Bartolazzi A, Peach R, Aruffo A, et al. Interaction between CD44 and hyaluronate is directly implicated in the regulation of tumor development. F Exp Med 1994;180:53-66.

78 Zeng C, Toole BP, Kinney SD, et al. Inhibition of tumor growth in vivo by hyaluronan oligomers. Int $\mathcal{F}$ Cancer 1998; 77:396-401.

79 Yu Q, Toole BP, Stamenkovic I. Induction of apoptosis of metastatic mammary carcinoma cells in vivo by disruption 
of tumor cell surface CD44 function. 7 Exp Med 1997;186: 1985-96.

80 Henke C, Bitterman P, Roongta U, et al. Induction of fibroblast apoptosis by anti-CD 44 antibody: implications for the treatment of fibroproliferative lung disease. Am f Patho 1996;149:1639-50.

81 Ayroldi E, Cannarile L, Migliorati G, et al. CD44 (Pgp-1) inhibits CD3 and dexamethasone-induced apoptosis. Blood 1995;86:2672-8.

82 Bates RC, Elith CA, Thorne RF, et al. Engagement of variant CD44 confers resistance to anti-integrin antibodymediated apoptosis in a colon carcinoma cell line. Cell Adhes Commun 1998;6:21-38.

83 Galluzzo E, Albi N, Fiorucci S, et al. Involvement of CD44 variant isoforms in hyaluronate adhesion by human activated T cells. Eur f Immunol 1995;25:2932-9.

84 Griffioen AW, Horst E, Heider $\mathrm{KH}$, et al. Expression of CD44 splice variants during lymphocyte activation and tumor progression. Cell Adhes Commun 1994;2:195-200.

85 Gunthert U, Hofmann M, Rudy W, et al. A new variant of glycoprotein CD44 confers metastatic potential to rat carglycoprotein CD44 confers meta

86 Rudy W, Hofmann M, Schwartz-Albiez R, et al. The two major CD44 proteins expressed on a metastatic rat tumor cell line are derived from different splice variants: each one individually suffices to confer metastatic behavior. Cancer Res 1993;53:1262-8.

87 Seiter S, Arch R, Reber S, et al. Prevention of tumor metastasis formation by anti-variant CD 44 . F Exp Med 1993; 177:443-55.

88 Sleeman JP, Arming S, Moll JF, et al. Hyaluronateindependent metastatic behavior of CD44 variantexpressing pancreatic carcinoma cells. Cancer Res 1996;56: 3134-41.

89 Reeder JA, Gotley DC, Walsh MD, et al. Expression of antisense CD44 variant 6 inhibits colorectal tumor metastasis and tumor growth in a wound environment. Cancer Res 1998;58:3719-26.

90 Moll J, Schmidt A, van der Putten $\mathrm{H}$, et al. Accelerated immune response in transgenic mice expressing rat CD44v4-v7 on T cells. f Immunol 1996;156;2085-94.

91 Driessens MH, Stroeken PJ, Rodriguez Erena NF, et al. Targeted disruption of CD44 in MDAY-D2 lymphosarcoma cells has no effect on subcutaneous growth or metastatic capacity. F Cell Biol 1995;131:1849-55.

92 Givehchian M, Worner S, Strater J, et al. No evidence for cancer-related CD44 splice variants in primary and metastatic colorectal cancer. Eur F Cancer 1998;34:1099104

93 Zahalka MA, Okon E, Gosslar U, et al. Lymph node (but not spleen) invasion by murine lymphoma is both CD44- and hyaluronate-dependent. F Immunol 1995;154:5345-55.

94 Matsumura Y, Tarin D. Significance of CD44 gene products for cancer diagnosis and disease evaluation [see comments]. Lancet 1992;340:1053-8.

95 Goodison S, Tarin D. Clinical implications of anomalous CD44 gene expression in neoplasia [in process citation]. Front Biosci 1998;3:E89-109.

96 Woodman AC, Sugiyama M, Yoshida K, et al. Analysis of anomalous CD 44 gene expression in human breast,
bladder, and colon cancer and correlation of observed bladder, and colon cancer and correlation of observed $\mathrm{mRNA}$

97 Sugino T, Gorham H, Yoshida K, et al. Progressive loss of CD44 gene expression in invasive bladder cancer. $A m ~ f$ Pathol 1996;149:873-82.
98 van Weering DH, Baas PD, Bos JL. A PCR-based method for the analysis of human CD44 splice products. PCR Methods Appl 1993;3:100-6.

99 Goodison S, Yoshida K, Sugino T, et al. Rapid analysis of distinctive CD44 RNA splicing preferences that characterize colonic tumors. Cancer Res 1997;57:3140-4.

100 Gorham H, Sugino T, Woodman AC, et al. Cellular distribution of CD44 gene transcripts in colorectal carcinomas and in normal colonic mucosa. F Clin Pathol 1996;49:4828.

101 Matsumura Y, Hanbury D, Smith J, et al. Non-invasive detection of malignancy by identification of unusual CD44 gene activity in exfoliated cancer cells. BMF 1994;308:61924 .

102 Matsumura Y, Sugiyama M, Matsumura S, et al. Unusual retention of introns in CD44 gene transcripts in bladder cancer provides new diagnostic and clinical oncological opportunities. F Pathol 1995;177:11-20.

103 Yoshida K, Bolodeoku J, Sugino T, et al. Abnormal retention of intron 9 in CD44 gene transcripts in human gastrointestinal tumors. Cancer Res 1995;55:4273-7.

104 Katoh S, McCarthy JB, Kincade PW. Characterization of soluble CD44 in the circulation of mice. Levels are affected by immune activity and tumor growth. If Immunol 1994;153:3440-9.

105 Ristamaki R, Joensuu H, Jalkanen S. Does soluble CD44 reflect the clinical behavior of human cancer? Curr Top Microbiol Immunol 1996;213:155-66.

106 De Rossi G, Marroni P, Paganuzzi M, et al. Increased serum levels of soluble CD44 standard, but not of variant isoforms v5 and v6, in B cell chronic lymphocytic leukemia. Leukemia 1997;11:134-41.

107 Bolodeoku J, Yoshida K, Sugino T, et al. CD44 expression in human breast cancer cell lines is related to oestrogen receptor (ER) status and confluency in vitro. Biochem Soc Trans 1997;25:356S

108 Goodison S, Yoshida K, Churchman M, et al. Multiple intron retention occurs in tumor cell CD44 mRNA processing. Am F Pathol 1998;153:1221-8.

109 Konig H, Moll J, Ponta H, et al. Trans-acting factors regulate the expression of CD44 splice variants. EMBO F 1996; 15:4030-9.

110 Konig H, Ponta H, Herrlich P. Coupling of signal ransduction to alternative pre-mRNA splicing by composite splice regulator. EMBO f 1998;17:2904-13.

111 Sugino T, Yoshida K, Zhao S, et al. Disorderly CD44 gene expression in human cancer cells can be modulated by growth conditions [in process citation]. F Pathol 1998;186: $17-23$.

112 Kogerman P, Sy MS, Culp LA. Over-expression of human CD44s in murine 3T3 cells: selection against during primary tumorigenesis and selection for during micrometastasis. Clin Exp Metastasis 1998;16:83-93.

113 Kogerman P, Sy MS, Culp LA. CD44 protein levels and its biological activity are regulated in Balb/c 3T3 fibroblasts by serum factors and by transformation with the ras but not with the sis oncogene. F Cell Physiol 1996;169:341-9.

114 Kogerman P, Sy MS, Culp LA. Oncogene-dependent expression of CD44 in Balb/c 3T3 derivatives: correlation with metastatic competence. Clin Exp Metastasis 1996;14: $73-82$. 